\title{
AVALIAÇÃO NEUROPSICOLÓGICA DE CRIANÇAS COM TRANSTORNO DO DÉFICIT DE ATENÇÃO E HIPERATIVIDADE (TDAH) REVISÃO DA LITERATURA
}

\author{
Gilda Maria Albaricci Nex ALVES ${ }^{1}$ \\ Carmen Maria Bueno NEME ${ }^{2}$ \\ Mirella Faraco CARDIA ${ }^{3}$
}

RESUMO: Este estudo apresenta uma revisão da literatura sobre a avaliação neuropsicológica de crianças com Transtorno do Déficit de Atenção e Hiperatividade (TDAH). Foram pesquisadas publicações nacionais indexadas nas bases de dados LILACS, SCIELO e Portal Capes disponíveis on line referentes ao período de 2000 a 2012. Os resultados indicaram um aumento da produção científica na área da avaliação neuropsicológica do transtorno de déficit de atenção. Contudo, observou-se a presença de poucos estudos nacionais que utilizam medidas consistentes de avaliação neuropsicológica, indicando a necessidade de pesquisas na área, especialmente voltadas às necessidades da prática da avaliação neuropsicológica.

PALAVRAS-CHAVE: Avaliação neuropsicológica. Avaliação psicológica. Transtorno do déficit de atenção e hiperatividade.

\section{Introdução}

O Transtorno de Déficit de Atenção/Hiperatividade (TDAH) caracteriza-se pela combinação dos sintomas de déficit de atenção, hiperatividade e impulsividade. Conforme Bolfer (2009), a incidência do TDAH em crianças e adolescentes de acordo com vários estudos realizados em diferentes regiões e culturas tanto no Brasil quanto internacionalmente, varia de 3 a 6\%, determinando prejuízos no âmbito familiar, escolar e social, bem como no desempenho acadêmico e no desenvolvimento emocional e afetivo.

De acordo Azevedo, Caixeta e Mendes (2009), o portador de TDAH demonstra um padrão de desatenção e/ou hiperatividade mais frequente do que o observado em

1 Psicóloga cursando mestrado em Psicologia do Desenvolvimento e Aprendizagem. UNESP Universidade Estadual Paulista. Supervisora do Centro de Reabilitação SORRI-BAURU. Bauru - SP Brasil. 17033-360 - gildaalbaricci@ig.com.br.

2 Livre Docente em Psicologia Clínica na UNESP. Pós-Doutorado no Laboratório de Estudos Psicofisiológicos do Stress da PUC-Campinas. Docente no Programa de Mestrado em Psicologia do Desenvolvimento e Aprendizagem. UNESP - Universidade Estadual Paulista. Bauru - SP - Brasil. 17033-360 - cmneme@terra.com.br.

3 Psicóloga. Ampliatta - Instituto de Psicologia e Saúde. Bauru - SP - Brasil. 17017-332 mirellaneuropsi@gmail.com. 
crianças com mesmo nível desenvolvimental. Consequentemente, dificulta a realização de processos que envolvam esforço mental por determinado período de tempo.

O diagnóstico do TDAH é fundamentalmente clínico, baseando-se em critérios operacionais claros e bem definidos, provenientes de sistemas classificatórios como o Diagnostic and Statistical Manual of Mental Health Disorder, Fouth Edition, 2000 (DSM-IV) e Classificação Internacional de Doenças, 10a edição, CID-10 (ARAÚJO, 2002). O TDAH pode ser subdividido segundo a presença ou ausência de hiperatividade / impulsividade e/ou déficit atencional, de acordo com os critérios do DSM-IV, que o classifica como combinado, predominantemente desatento e predominantemente hiperativo/impulsivo.

Os critérios do DSM IV envolvem a persistência em mais de um contexto, ocorrer antes dos sete anos e duração mínima de seis meses de comportamentos que envolvem desatenção-hiperatividade-impulsividade. A desatenção se manifesta por facilidade em distrair-se com estímulos alheios à tarefa, esquecimento em atividades diárias, distração durante conversas, desatenção ou não cumprimento de regras em atividades lúdicas, alternância constante de tarefas, além de evitar tarefas complexas e que exijam organização. A hiperatividade caracteriza-se pela movimentação e fala excessivas, dificuldade de ficar sentado e tendência a correr ou escalar em situações inapropriadas A impulsividade envolve o agir sem pensar e dificuldade em esperar sua vez em atividades lúdicas ou em situações de grupo.

De acordo com Santos e Vasconcelos (2010), o diagnóstico deve ser refeito a cada semestre, sugerindo aspecto dinâmico e transitório do transtorno. Os autores apontam a relação entre desatenção, hiperatividade e impulsividade, no transcorrer dos últimos seis meses podendo resultar em diferentes subtipos de diagnóstico de TDAH e propõe a marcação temporal de seis meses para acompanhamento.

O TDAH pode manifestar-se isoladamente apesar da alta incidência de comorbidades, isto é, a simultaneidade de ocorrência de dois ou mais transtornos ou outros problemas orgânicos. Um quadro de TDAH acompanhado de comorbidades pode dificultar o diagnóstico preciso. De acordo com Biederman et al. (apud ROHDE; HALPERN, 2004), as pesquisas mostram uma alta prevalência de comorbidade entre o TDAH e coloca dados estatísticos como os transtornos de conduta e opositor desafiante situada em torno de 30 a 50\%. A taxa de comorbidade também é significativa com depressão (15 a 20\%); transtornos de ansiedade (25\%) e transtornos da aprendizagem (10 a 25\%). 
Bolfer (2009) destaca que as causas desse transtorno, apesar de ainda não completamente definidas, podem ser atribuídas a uma combinação de fatores genéticos, biológicos e ambientais. A autora cita em seu estudo, pesquisas neuropsicológicas e de neuroimagem indicando comprometimento de funções do córtex pré-frontal nos portadores de TDAH e ressalta a importância dessa região em relação aos sintomas apresentados, complementa ainda, destacando pesquisas de imagem cerebral que demonstram áreas de tamanho reduzido do córtex pré-frontal, núcleos de base e cerebelo em pacientes com TDAH, discorrendo sobre as múltiplas projeções de neurônios ricos em dopamina e noradrenalina, serotoninas, glutaminas e acetilcolinas que tais regiões recebem, reforçando os aspectos multi-determinantes do TDAH.

De acordo com Gonçalves et al. (2013), nos últimos anos, estudos apontam além das dificuldades do controle atencional e do grau de atividade e impulsos, evidências de prejuízos em funções executivas.

Pesquisas neuropsicológicas demonstram que crianças com TDAH podem ter mais dificuldades que seus pares sem o transtorno, em atividades que exijam inibição, atenção, automonitoramento e executivo central da memória de trabalho (COSTA; DORNELES; ROHDE, 2012). O autor relaciona tais evidências com demais achados em pesquisas que atribuem ao déficit inibitório com uma das causas das dificuldades atencionais e executivas de crianças com TDAH. Destaca que situações que exigem automonitoramento e inibição da resposta, aspectos incluídos nas funções executivas, necessitam de adequada capacidade do componente memória de trabalho para serem executadas.

Bolfer (2009) ressalta que esta característica pode ser observada na criança através de suas respostas antes do término da pergunta ou na dificuldade em conseguir sustentar a atenção diante de um estímulo externo importante. Os estudos da autora atribuem a falha no controle inibitório e a não sustentação da atenção como a causa do déficit cognitivo, da recepção desorganizada da informação e das falhas no armazenamento da informação.

Considerando a complexidade das diversas variáveis que envolvem o TDAH, evidencia-se a importância de uma avaliação minuciosa e específica deste transtorno. Gonçalves et al. (2013) conclui que a avaliação neuropsicológica ainda não é plenamente incluída nos protocolos de avaliação clinica e nos estudos científicos. Descreve como essa avaliação busca identificar e descrever os padrões de funcionamento cognitivo típico, esperado de acordo com o nível sociocultural, 
desenvolvimental e outras diferenças individuais da população e destaca que tal avaliação pode permitir caracterizar as funções cognitivas com desempenho preservado e aquelas com desempenho prejudicado.

Rohde e Halpern (2004) ressaltam a importância da integração dos processos de investigação, os quais devem considerar os dados obtidos junto aos pais, professores e a própria criança. Propõe que a consideração isolada de uma única fonte de informações pode ser prejudicada pelos dados fornecidos pela família e pela tendência dos professores em maximizarem os problemas de comportamento. Em contrapartida, a avaliação baseada apenas em técnicas de observação e exame clínico, de acordo com o autor, pode não ser capaz de identificar alguns marcadores de TDAH. Em certas situações, as crianças e adolescentes conseguem controlar seus sintomas temporariamente através de esforço e envolvimento voluntário em atividades que lhe despertem interesse. Por tais razões, a abordagem interdisciplinar e a utilização de recursos variados para diagnóstico e intervenções voltadas ao TDAH são recomendadas expressamente por diversos especialistas da área (REPPOLD, 2005).

Reppold (2005) cita que através dessa proposta, é possível analisar comportamentos disfuncionais previamente identificados pelas escalas objetivas relacionando-os ao contexto de vida do sujeito, descreve que nem todas as indicações de alteração são evidências diagnósticas, uma vez que a presença de indicadores de desatenção e hiperatividade/impulsividade pode ser decorrente de uma alteração afetiva ou de uma situação estressora e, portanto, não preencher necessariamente critérios para TDAH.

Nesta mesma direção, os Manuais voltados a critérios para diagnóstico, como o DSM IV, enfatizam como considerações no diagnóstico do TDAH, que os comportamentos como desorganização, dificuldades escolares e dificuldade em cumprir combinados devem relacionar-se a resultados de alterações em processos cognitivos como atenção, memória e não decorrentes de características de personalidade ou consequência exclusiva de outras disfunções psicológicas, como um padrão de comportamento deliberadamente oposicionista ou desafiador, dependência de substâncias, transtornos de aprendizagem ou deficiência intelectual.

Assim, considerando a importância da avaliação neuropsicológica infantil para a investigação clínica de transtornos psicológicos, este estudo apresenta uma revisão da literatura sobre o tema e descreve os instrumentos neuropsicológicos utilizados nesta área. 


\section{Objetivos}

Identificar e descrever artigos nacionais publicados sobre avaliação neuropsicológica de crianças com Transtorno do Déficit de Atenção e Hiperatividade (TDAH) nas bases de dados LILACS, SCIELO e Portal Capes, disponíveis on-line e publicadas período de 2000 a 2012.

\section{Método}

Este estudo caracteriza-se por uma revisão de literatura. Trata-se de uma forma de pesquisa que utiliza como fonte de dados a literatura sobre o tema avaliação neuropsicológica de crianças com Transtorno do Déficit de Atenção e Hiperatividade (TDAH).

As bases de dados escolhidas para a pesquisa de artigos consistiu dos bancos de dados LILACS, SCIELO e Portal Capes disponíveis on-line, selecionando-se trabalhos publicados de 2000 a 2012. Foram adotados também os critérios de inclusão: os artigos deveriam citar instrumentos de avaliação psicológica e neuropsicológica e a faixa etária dos sujeitos das pesquisas deveria estar entre 6 e 17 anos de idade. Foram adotados os seguintes critérios de exclusão: relatos de casos de TDAH em comorbidade com outros diagnósticos e indisponibilidade dos artigos científicos na íntegra.

Foram utilizadas as palavras-chave "avaliação neuropsicológica", "avaliação psicológica", "transtorno do déficit de atenção e hiperatividade", combinando dois termos.

Os resumos obtidos nos bancos de dados foram comparados entre si para a verificação de superposição de artigos, excluindo-se os artigos repetidos. Posteriormente, os resumos foram analisados para selecionar trabalhos que atendessem aos critérios de inclusão. Nesta fase de coleta de dados, foram selecionados resumos e uma nova etapa de apreciação dos artigos completos permitiu chegar à definição final dos textos que foram analisados neste estudo.

Os artigos completos foram examinados segundo os critérios adotados no presente estudo, excluindo-se os que não os atendiam.

Os registros dos dados coletados foram analisados considerando-se os aspectos: 1) ano de publicação, 2) tipo de estudo, 3) objetivos do estudo, 4) idade da população, 5) gênero, 6) número de participantes, 7) instrumentos de avaliação utilizados. 


\section{Resultados e discussão}

Foram obtidos sete artigos que atenderem aos critérios de elegibilidade para o estudo.

O quadro 1 apresenta a distribuição dos artigos de acordo com o ano de publicação, sendo possível observar um maior número de publicações nos anos de 2008 a 2012. Contudo, pode-se considerar que ainda são poucas as pesquisas nacionais desenvolvidas sobre o tema e acessíveis on-line.

Quadro 1 - Distribuição dos artigos publicados entre 2000 e 2012

\begin{tabular}{|c|c|}
\hline Ano & Número de publicações \\
\hline 2000 & 1 \\
\hline 2001 & 1 \\
\hline 2008 & 1 \\
\hline 2009 & 2 \\
\hline 2010 & 1 \\
\hline 2012 & 7 \\
\hline total & 1 \\
\hline
\end{tabular}

Fonte: Elaborado pelas autoras.

A escassa produção científica voltada à temática da avaliação neuropsicológica do TDAH pode gerar diagnósticos incorretos, conforme citado por Reppold (2005).

A população estudada em todos os artigos científicos que compuseram este estudo foi composta por meninos e meninas, não se encontrando referência a diferenças entre gêneros, embora, na literatura específica seja citada com maior frequência o TDAH em meninos (ROHDE; HALPERN, 2004).

Dos sete artigos selecionados, três descreveram estudos com população envolvendo crianças, sendo dois deles com população inferior a trinta participantes e um deles com população acima de duzentos sujeitos. Os demais artigos referem-se ao levantamento de dados com professores, pais e profissionais sobre o tema. O quadro 2 apresenta dados sobre a população abordada nos estudos. 
Quadro 2 - Distribuição da frequência dos participantes nos artigos científicos com população de crianças

\begin{tabular}{|c|c|}
\hline Participantes & Frequência \\
\hline $\begin{array}{c}10 \text { crianças com queixas de comportamento e } \\
10 \text { crianças do grupo controle }\end{array}$ & 1 \\
\hline 255 crianças & 1 \\
\hline 30 crianças & 1 \\
\hline 277 pais e 282 professores & 1 \\
\hline $\begin{array}{c}10 \text { neurologistas, } 10 \text { psiquiatras e } 10 \\
\text { psicólogos }\end{array}$ & 1 \\
\hline 136 professores & 1 \\
\hline 30 crianças & \\
\hline
\end{tabular}

Fonte: Elaborado pelas autoras.

Quanto ao diagnóstico de TDAH, os dados deste estudo revelaram que entre as pesquisas publicadas no período de 2000 a 2012, três utilizaram apenas entrevistas, escalas ou anamnese, aplicados em pais e professores. Um dos estudos utilizou apenas testes neuropsicológicos e três deles utilizaram testes, entrevistas e observação da criança em situações lúdicas ou de interação.

A avaliação neuropsicológica tem como objetivo estudar as relações entre a atividade cerebral, cognição e o comportamento. De acordo com Lezak, Howieson e Loring, (2004), considera as alterações cognitivas, comportamentais e emocionais de acordo com a natureza, extensão e localização da lesão cerebral, além da influência das variáveis idade, gênero, condições físicas e contexto psicossocial de desenvolvimento.

Borges et al. (2008) descreve sobre o uso restrito e recente da avaliação neuropsicológica para os quadros psicopatológicos da infância e adolescência e sobre a lacuna na literatura brasileira no que se refere a este tema.

Ainda quanto aos instrumentos de avaliação, verificou-se que o teste WISC III Escala de Inteligência Wechsler para crianças foi utilizado em dois artigos. Em duas pesquisas foram utilizados instrumentos para medida das funções atencionais, sendo citados: Teste de Cancelamento (TC), Color Trail Test (CTT), Teste de Atenção por Cancelamento (TAC) e Teste de Trilhas - parte B. E investigação do desempenho 
escolar foi realizada apenas em um dos estudos, através do TDE: teste de desempenho escolar.

Apenas um dos artigos analisados, utilizou o teste Wisconsin de Classificação de Cartas e Evocação Seriada reversa para números, sendo o primeiro um instrumento para Avaliação psicológica e Neuropsicológica adaptado e padronizado para uso no Brasil e usado como uma medida de flexibilidade mental e o segundo voltado a funções executivas e avaliação da memória operacional. Estudos neuropsicológicos sugerem que as limitações em quadros de TDAH podem ser verificadas em mais de um domínio cognitivo. As funções mais associadas ao possível prejuízo cognitivo relacionado ao TDAH são atenção, memória de trabalho e componentes de funções executivas (GONÇALVES et al., 2013), justificando a importância de aprofundamento na avaliação psicológica. Dos sete artigos selecionados para este estudo, dois citaram os critérios diagnósticos do DSM IV como um dos instrumentos utilizados na avaliação.

A figura 1 representa a informação referente ao profissional que realizou as avaliações citadas nos artigos científicos. Cinco artigos (71\%) informaram a participação de psicólogos e dois deles (29\%) citaram psicólogos em conjunto com profissional da área de medicina.

Figura 1 - Profissão dos pesquisadores

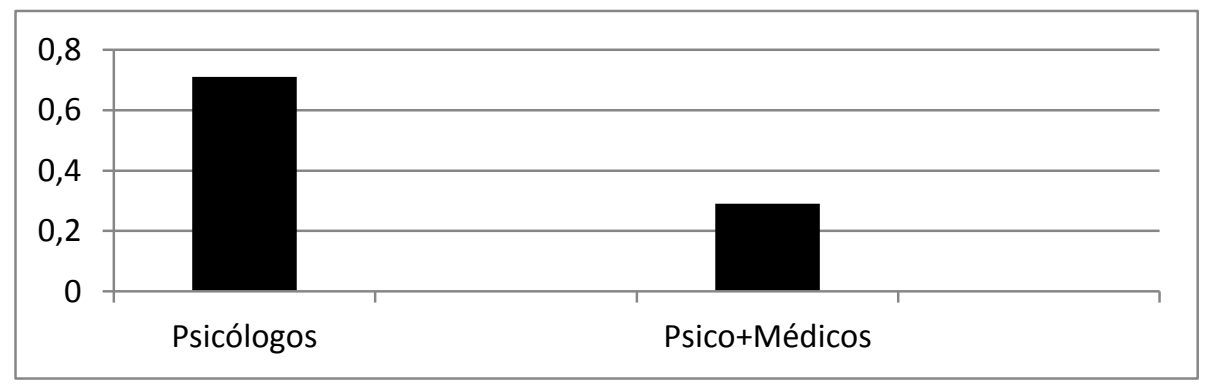

Fonte: Elaborada pelas autoras.

Evidencia-se a participação expressiva de psicólogos nesta área de pesquisa e a crescente parceria desenvolvida com médicos, o que é bastante benéfico para se construir diálogos mais produtivos entre estes profissionais, com ganhos para pacientes.

\section{Considerações finais}


A partir dos resultados encontrados neste estudo, constata-se que as publicações sobre o TDAH vêm aumentando nos últimos anos, progressivamente, entretanto o número de publicações brasileiras ainda é escasso.

Destaca-se a importância da avaliação neuropsicológica, que pode contribuir para um maior conhecimento sobre as relações entre prejuízos cognitivos e psicopatologia.

Amostras muito específicas e a utilização de instrumentos reduzidos são algumas das limitações que podem comprometer a generalização dos resultados. Dessa forma, observa-se que são necessários cuidados metodológicos para que os estudos sobre prejuízos neuropsicológicos na infância sejam mais consistentes.

Como o TDAH interfere nas atividades diárias e escolares, os profissionais da área da educação e da saúde devem estar preparados para a avaliação e reabilitação destas crianças, de forma a minimizar o impacto na qualidade de vida e interação social desta população.

\title{
CHILDREN NEUROPSYCHOLOGICAL ASSESSMENT ATTENTION-DEFICIT HYPERACTIVITY DISORDER (ADHD) LITERATURE REVIEW
}

\begin{abstract}
This study presents a review of literature on the neuropsychological assessment of children with Attention Deficit Disorder and Hyperactivity Disorder (ADHD). Indexed national publications were searched in LILACS, SciELO and Capes Portal available on line pertaining to the period 2000-2012. Database the results indicated an increase in scientific production in the neuropsychological evaluation of attention deficit disorder. However, the presence of few national studies that use consistent measures of neuropsychological assessment was observed, indicating the need for research in this area, especially geared to the needs of the practice of neuropsychological assessment.
\end{abstract}

KEYWORDS: Neuropsychological assessment. Psychological assessment disorder. Attention deficit hyperactivity disorder.

\section{REFERÊNCIAS}

ARAÚJO, A. P. Q. C. Avaliação e manejo de crianças com dificuldade escolar e distúrbio de atenção. Jornal de Pediatria, Rio de Janeiro, v.78, supl.1, p.104-110, 2002.

AZEVEDO, P. V. B.; CAIXETA, L. F.; MENDES, G. M. Estudos epidemiológicos em neuropsiquiatria infantil com ênfase no transtorno de déficit de atenção e hiperatividade. Revista Brasileira de Neurologia, Rio de Janeiro, v.45, n.4, p.35-40, 2009. 
BOLFER, C. P. M. Avaliação neuropsicológica das funções executivas e da atenção em crianças com transtorno do déficit de atenção/hiperatividade (TDAH). 2009.

123 f. Dissertação (Mestrado em Ciências) - Faculdade de Medicina da Universidade de São Paulo, São Paulo, 2009.

BORGES, J. L. et al. Avaliação neuropsicológica dos transtornos psicológicos na infância: um estudo de revisão. Psico-USF (Impr.), Itatiba, v.13, n.1, 2008.

Disponível em: <http://www.scielo.br/scielo.php?script=sci_arttext\&pid=S141382712008000100015\&lng=en\&nrm=iso>. Acesso em: 09 mar. 2014.

COSTA, A. C.; DORNELES, B. V.; ROHDE, L. A.P. Identificação dos procedimentos de contagem e dos processos de memória em crianças com TDAH. Psicologia:

Reflexão e Crítica, Porto Alegre, v.25, n.4, p.791-801, 2012.

GONÇALVES, H. A. et al. Componentes atencionais e de funções executivas em meninos com TDAH: dados de uma bateria neuropsicológica flexível. Jornal Brasileiro de Psiquiatria, Rio de Janeiro, v.62, n.1, 2013.

LEZAK, M. D.; HOWIESON, D. B.; LORING, D. W. Neuropsychological assessment. 4.ed. New York: Oxford University Press, 2004.

REPPOLD, C. T. Construção, validação e normatização de uma bateria de cinco escalas para avaliação de ajustamento psicológico em adolescentes. 2005. $334 \mathrm{f}$. Tese (Doutorado em Psicologia) - Universidade Federal do Rio Grande do Sul, Rio Grande do Sul, 2005.

ROHDE, L. A.; HALPERN, R. Transtorno de déficit de atenção/ hiperatividade: atualização. Jornal de Pediatria, Rio de Janeiro, v.80, n.2, p.61-70, 2004.

SANTOS, L. F.; VASCONCELOS L. A. Transtorno do déficit de atenção e hiperatividade em crianças: uma revisão interdisciplinar. Psicologia: Teoria e Pesquisa, Brasília, v.26, n.4, p.717-24, 2010. 